Recepción: 17 / 06 / 2019

Aceptación: 12 / 07 / 2019

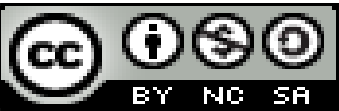

Ciencias económicas y empresariales

Publicación: 05 / 08 / 2019

Artículo de investigación

\title{
Técnicas del aprendizaje acelerado en la enseñanza de las estadísticas
}

Accelerated learning techniques in teaching statistics

Técnicas de aprendizagem acelerada no ensino de estatística

Homero Manuel Ferrin-Schettini I homeromanuelferrin@hotmail.com

Correspondencia: homeromanuelferrin@ hotmail.com

I. Doctor en Ciencias Administrativas, Diploma Superior en Economía Internacional, Diploma Superior en Economía del Ecuador y del Mundo, Diploma Superior en Gestión Empresarial Internacional, Magister en Negocios Internacionales y Gestión de Comercio Exterior, Ingeniero Civil, Arquitecto, Docente de la Universidad Laica Eloy Alfaro de Manabí, Manta, Ecuador. 


\title{
Resumen
}

Las técnicas de aprendizaje acelerado aplicadas a los alumnos permiten a los docentes de educación superior una intervención educativa sistemática que facilite a los estudiantes herramientas intelectuales y sociales en pro de aprender a aprender como modelo centrado en el aprendizaje. El estudio evaluó el manejo de las técnicas del aprendizaje acelerado en la enseñanza de la estadística. Para ello, se utilizó una metodologia de tipo descriptivo y un diseño no experimental. La población la constituyeron 60 estudiantes que cursan materias del área de Estadísticas en la Universidad Laica Eloy Alfaro de Manabí (ULEAM). La recolección de la información fue a través de un instrumento tipo cuestionario, con una escala tipo lickerd con 3 alternativas de repuestas. La validez del instrumento fue realizada a través del criterio de expertos en el área, y la confiabilidad se determinó a través del Coeficiente de Alfa Cronbach, cuyo resultado fue de 0.87, considerado altamente significativo. La técnica de análisis fue la estadística descriptiva y frecuencial. Los resultados del análisis permitieron concluir que la música como técnica del aprendizaje acelerado en la enseñanza de las estadísticas, alcanzo el mayor uso entre los docentes, implicando que sirve como medio para equilibrar la mente racional y la emocional de sus estudiantes.

Palabras Clave: técnicas del aprendizaje acelerado; música; mapas conceptuales y relajación y la atención.

\begin{abstract}
Accelerated learning techniques applied to students allow higher education teachers a systematic educational intervention that provides students with intellectual and social tools for learning to learn as a model focused on learning. The study evaluated the management of accelerated learning techniques in the teaching of statistics. For this, a descriptive methodology and a non-experimental design were used. The population was constituted by 60 students who study subjects in the area of Statistics at the Eloy Alfaro de Manabí Lay University (ULEAM). The information was collected through a questionnaire type instrument, with a lickerd scale with 3 alternative answers. The validity of the instrument was performed through the criteria of experts in the area, and the reliability was determined through the Alpha Cronbach Coefficient, whose result was 0.87, considered highly significant. The analysis technique was descriptive and frequency statistics. The results of the analysis allowed us to conclude that music as a technique of accelerated learning in the teaching of statistics,
\end{abstract}


reached the greatest use among teachers, implying that it serves as a means to balance the rational and emotional mind of their students.

Keywords: accelerated learning techniques; music; Concept maps and relaxation and attention.

\section{Resumo}

Técnicas de aprendizagem aceleradas aplicadas aos alunos permitem aos professores do ensino superior uma intervenção educacional sistemática que fornece aos alunos ferramentas intelectuais e sociais para aprender a aprender como um modelo focado na aprendizagem. O estudo avaliou a gestão de técnicas de aprendizagem acelerada no ensino de estatística. Para isso, utilizou-se metodologia descritiva e delineamento não experimental. A população foi constituída por 60 alunos que estudam disciplinas na área de Estatística da Universidade Eloy Alfaro de Manabí Lay (ULEAM). As informações foram coletadas por meio de um instrumento do tipo questionário, com uma escala de lickerd com 3 respostas alternativas. A validade do instrumento foi realizada por meio de critérios de especialistas da área, e a confiabilidade foi determinada pelo Coeficiente de Cronbach Alfa, cujo resultado foi de 0,87 , considerado altamente significativo. A técnica de análise foi descritiva e estatística de frequência. Os resultados da análise permitiram concluir que a música como técnica de aprendizagem acelerada no ensino de estatística, alcançou o maior uso entre os professores, implicando que ela serve como um meio de equilibrar a mente racional e emocional de seus alunos.

Palavras-chave: técnicas de aprendizagem acelerada; música; Mapas conceituais e relaxamento e atenção.

\section{Introducción}

Las técnicas o estrategias de aprendizaje acelerado incrementan el potencial de aprendizaje en las aulas y una vez que los alumnos hayan salido de las mismas, es decir pueden ser utilizadas a lo largo de la vida; pueden adaptarse a la construcción individual y colaborativa del conocimiento; y ser aprendidas no importando la edad de los alumnos, ya que si bien es deseable que se tenga acceso a este tipo de aprendizaje desde la infancia y juventud, la mayoría de las técnicas de aprendizaje acelerado también pueden ser utilizadas de manera efectiva para el aprendizaje de adultos, incluyendo los de la tercera edad y en todo tipo de organizaciones.

Es de indicar, que esta técnica a diferencia de otras presentes es sumamente amigable para el cerebro. El aprendizaje acelerado sigue un enfoque multisensorial y multimodal, utilizando para ello una multiplicidad de inteligencias con las que los estudiantes pueden acceder al conocimiento. El modelo de aprendizaje acelerado 
utiliza también las emociones y la reflexión para lograr el aprendizaje. Todos estos elementos convierten al modelo de aprendizaje acelerado en un modelo metacognitivo. En el caso particular de este artículo, se aplicaron las técnicas de aprendizaje acelerado con la finalidad de evaluar su efectividad en la comprensión con mayor facilidad de los contenidos programáticos del área de estadística.

\section{Desarrollo}

\subsection{La relajación y la atención}

Drapeau (1996), señala la relajación como una técnica fundamental del aprendizaje acelerado ya que cuando se manifiesta alguna tensión, las capacidades de aprendizaje y la aptitud para realizar cualquier trabajo de tipo intelectual se hallan afectadas. De allí que se utiliza para alcanzar los "estados de conciencia" durante los cuales el cerebro funciona con mayor agudeza, intensidad y eficacia. Toda visualización hecha en estados alterados de conciencia tendrá una repercusión asombrosa. Toda información recibida será comprendida e integrada con mejores resultados. Saber crear en el organismo un estado de gran receptividad permite recibir más fácilmente la información, comprenderla en profundidad y asimilarla para retenerla mejor y así poder acceder a ella cuando se requiera, a voluntad. Sus beneficios se pueden estudiar a partir de las ondas cerebrales y la atención. (Pisano, B. 2000).

Las ondas cerebrales: dado que existen numerosos planos de conciencia a través de los cuales fluctuamos de acuerdo a nuestras actividades y disposición interior.

La atención: el coeficiente de retención de una información es directamente proporcional a la atención prestada en el momento de recibir dicha información. Con frecuencia las personas no atienden lo suficiente cuando se les presenta una información y en consecuencia no la captan e integran correctamente.

\subsection{Utilización de la música}

En aprendizaje acelerado es importante encontrar el equilibrio entre la mente racional y la mente emocional; para tal efecto, se propone un nuevo paradigma educativo, en la que el arte juega un fundamental rol, de allí que la utilización de la música ha sido una de las técnicas utilizadas en el aprendizaje acelerado en el aula de clases. Drapeau (1996), señala que la música actúa sobre nuestra psique de manera innegable, pero también influye sobre el cuerpo, a nivel orgánico. También se ha demostrado la influencia de la música sobre la tensión muscular, la respiración, la digestión y el ritmo 
cardiaco. Por ello, para entender los efectos benéficos de la música para el aprendizaje es necesario de manera somera hacer mención a dos elementos de la música: el ritmo y la tonalidad o la frecuencia, sin dejar de mencionar que la melodía, la armonía y el timbre afectan de manera importante las emociones.

Pulpillo (2001), explica que entre los beneficios que presta durante el aprendizaje, en pro de del desarrollo humano durante su utilización se destacan:

- Enriquecer su vida ayudándoles a profundizar en su mundo emocional y en el de los demás.

- Tener un soporte emocional frente a los avatares de su vida, constituyendo un medio de consuelo para la soledad o la incomprensión.

- Convertir la música en una afición.

- Despertar el gusto por la música de buena calidad porque es una de las herencias más preciadas de la humanidad.

- Afiliarse dentro de ellos mismos, hacerlos radicalmente interiores, despertando lo mejor, lo más noble de su vida: el amor a las personas y al trabajo que realizan como medio para alcanzar su proyecto de vida.

No obstante, la importancia de la música para el aprendizaje particular de la estadística, estarían dado en aumentar el entusiasmo de los alumnos por aprender la materia de esta forma.

\subsection{Mapas Mentales}

Buzan (2004), define los mapas mentales como método de análisis y como técnica gráfica: "Un mapa mental es un método de análisis que permite organizar con facilidad los pensamientos y utilizar al máximo las capacidades mentales" (p.26). Del mismo modo, indica que es " una expresión del pensamiento irradiante y, por tanto, una función natural de la mente humana. Es una poderosa técnica gráfica que nos ofrece una llave maestra para acceder al potencial del cerebro" (p 69)

Asimismo, la base de los mapas mentales es el pensamiento irradiante, que se puede considerar la pauta de pensamiento del cerebro humano. Buzan (2004), señala que, con la expresión de pensamiento irradiante, de irradiar, "en el sentido de dispersarse o moverse en diversas direcciones a partir de un centro determinado", se hace referencia "a aquellos procesos de pensamiento asociativos que proceden de un punto central o se conectan con él”. (p 69). Se destacan 4 características, a saber: 
a.- El tema motivo de atención, se constituye en una imagen central.

b.-Los principales aspectos del tema irradian de la imagen central de forma ramificada.

c.-Las ramas comprenden una imagen o una palabra clave impresa sobre una línea asociada. Los aspectos de menor importancia también están representados como ramas adheridas a las ramas de nivel superior.

d.- Las ramas forman una estructura conectada.

De acuerdo con estas características, un mapa mental además de que permite incluir una considerable cantidad de información en una sola página, también permite mostrar las relaciones existentes entre los conceptos e ideas. Para jerarquizar y categorizar durante la elaboración de los mapas mentales, plantea Cervantes (1999), es necesario identificar las ideas ordenadoras básicas, es decir de conceptos básicos para hacer posible la organización de otros conceptos.

\section{Metodologia}

La investigación se ubicó en un estudio descriptivo y no experimental, de acuerdo a Hernández, Fernández y Baptista (2014), afirman que la investigación descriptiva es la que se orienta a redactar informes relacionados con el estado real de las personas, objeto situaciones o fenómenos tal cual se presentan en el momento de su recolección, describe lo que mide sin analizar inferencias ni verificar hipótesis. La población la constituyeron 60 estudiantes activos que cursan la materia del área de estadística en la Universidad Laica Eloy Alfaro de Manabí (ULEAM). La recolección de la información fue a través de un instrumento tipo cuestionario, con una escala tipo lickerd con 3 alternativas de repuestas. La validez del instrumento fue realizada a través del criterio de expertos en el área y la confiabilidad se determinó a través del Coeficiente de Alfa Cronbach, cuyo resultado fue de 0.87 , considerado altamente significativo. La técnica de análisis fue la estadística descriptiva y frecuencial. 


\section{Resultados}

En el análisis de los resultados posterior a la aplicación del instrumento que permitió obtener la información relacionada a las técnicas del aprendizaje acelerado en la enseñanza de las estadísticas, en la que se pudo constatar:

Tabla $\mathbf{N}^{\mathbf{0}} 1$. Distribución frecuencial y porcentual del indicador relajación y la atención

\begin{tabular}{ccccc}
\cline { 2 - 5 } & \multicolumn{4}{c}{ ESTUDIANTES } \\
\hline ANÁLISIS/ ALTERNATIVAS & $\boldsymbol{F R}$ & $\boldsymbol{\%}$ & $\boldsymbol{F R A}$ & $\boldsymbol{\%} \boldsymbol{A}$ \\
\hline SIEMPRE & 7 & 12 & 7 & 12 \\
\hline ALGUNAS VECES & 32 & 53 & 39 & 65 \\
\hline NUNCA & 21 & 35 & 60 & 100 \\
\hline TOTAL & 60 & 100 & 60 & 100 \\
\hline
\end{tabular}

Fuente: Elaboración propia (2019).

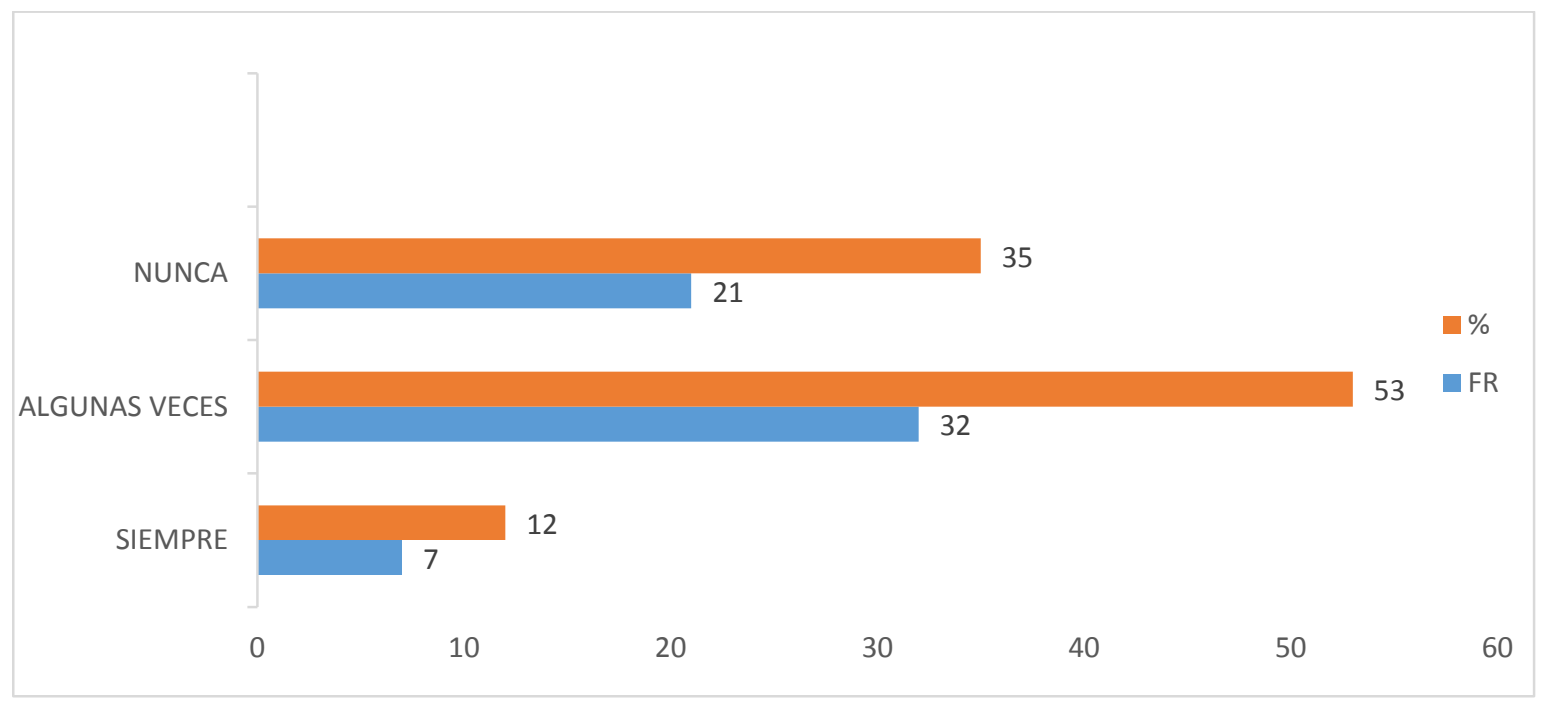

Gráfico $\mathbf{N}^{\mathbf{0} 1}$. Distribución frecuencial y porcentual del indicador relajación y la atención

Fuente: Elaboración propia (2019). 
En la tabla y gráfico $\mathrm{N}^{\mathrm{0}}$ 1, se presenta la distribución frecuencial y porcentual del indicador relajación y la atención como técnicas del aprendizaje acelerado en la enseñanza de las estadísticas, en la que se evidencio que el 53\% de los investigados coincidieron en opinar que solo algunas veces se utiliza la técnica de relajación y la atención de manera que se pueda crear en cada uno de los estudiantes un estado de gran receptividad para recibir de forma fácil todos los conocimientos que en materia de estadística son necesario para la compleja actividad diaria del quehacer académico. Seguido del $35 \%$ que indicaron que nunca la utilizan los docentes y solo el $12 \%$ considero su uso total. Es de indicar que la relajación como una técnica fundamental del aprendizaje acelerado, ayuda a minimizar la manifestación de alguna tensión, ampliar las capacidades de aprendizaje y la aptitud para realizar cualquier trabajo de tipo intelectual que puedan ser afectados.

Tabla №2. Distribución frecuencial y porcentual del indicador utilización de la música

\begin{tabular}{ccccc}
\cline { 2 - 5 } & \multicolumn{4}{c}{ DOCENTES } \\
\hline ANÁLISIS/ ALTERNATIVAS & $\boldsymbol{F R}$ & $\boldsymbol{\%}$ & $\boldsymbol{F R A}$ & $\boldsymbol{\%} \boldsymbol{A}$ \\
\hline SIEMPRE & 14 & 23 & 14 & 23 \\
\hline ALGUNAS VECES & 30 & 50 & 44 & 73 \\
\hline NUNCA & 16 & 27 & 60 & 100 \\
\hline TOTAL & 60 & 100 & 60 & 100 \\
\hline
\end{tabular}

Fuente: Elaboración propia (2019). 


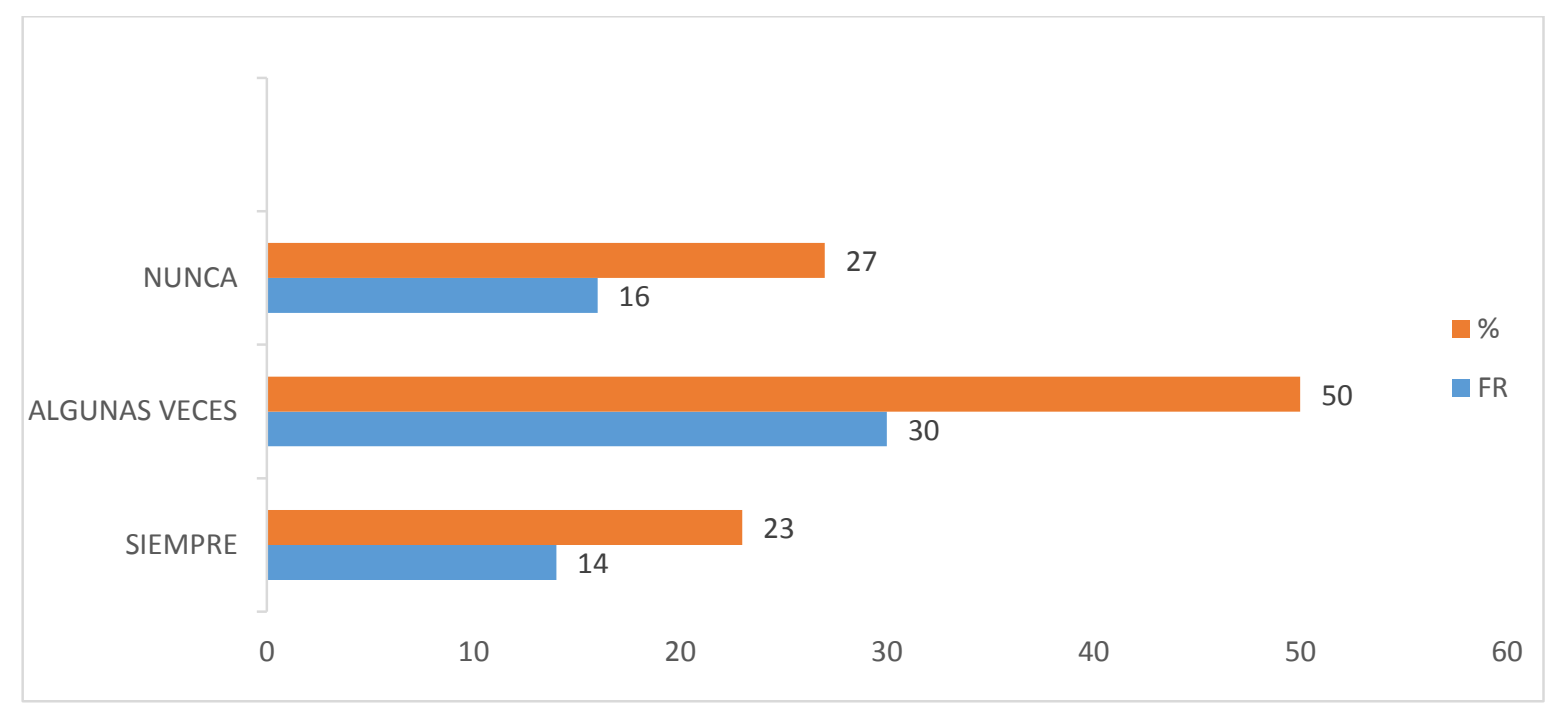

Tabla N². Distribución frecuencial y porcentual del indicador utilización de la música

Fuente: Elaboración propia (2019).

En la tabla y grafico No2, se presenta la distribución frecuencial y porcentual del indicador utilización de la música como técnica del aprendizaje acelerado en la enseñanza de las estadísticas, en la que se evidencio que el $50 \%$ de los investigados coincidieron en opinar que solo algunas veces se utiliza la técnica de la música como medio para equilibrar la mente racional y la emocional. Seguido del $27 \%$ que negaron esta posición para con. los docentes y el menor porcentaje se adjudicaron a la alternativa que más favorece la evaluación del indicador con solo el 23\% del total de las repuestas. En tal sentido, es de destacar el hecho en la cual se ha demostrado la influencia de la música sobre la tensión muscular, la respiración, la digestión y el ritmo cardiaco. Por ello, para entender los efectos benéficos de la música para el aprendizaje particular de la estadística, se deben combinar el ritmo y la tonalidad o la frecuencia, sin dejar de mencionar que la melodía, la armonía y el timbre para lograr un aprendizaje más acelerado. 
Tabla No 3. Distribución frecuencial y porcentual del indicador utilización de la música

\begin{tabular}{ccccc}
\cline { 2 - 4 } & \multicolumn{4}{c}{ DOCENTES } \\
\hline ANÁLISIS/ ALTERNATIVAS & FR & \% & FRA & $\%$ A \\
\hline SIEMPRE & 12 & 20 & 12 & 20 \\
\hline ALGUNAS VECES & 38 & 63 & 50 & 83 \\
\hline NUNCA & 10 & 17 & 60 & 100 \\
\hline TOTAL & 60 & 100 & 60 & 100 \\
\hline
\end{tabular}

Fuente: Elaboración propia (2019).

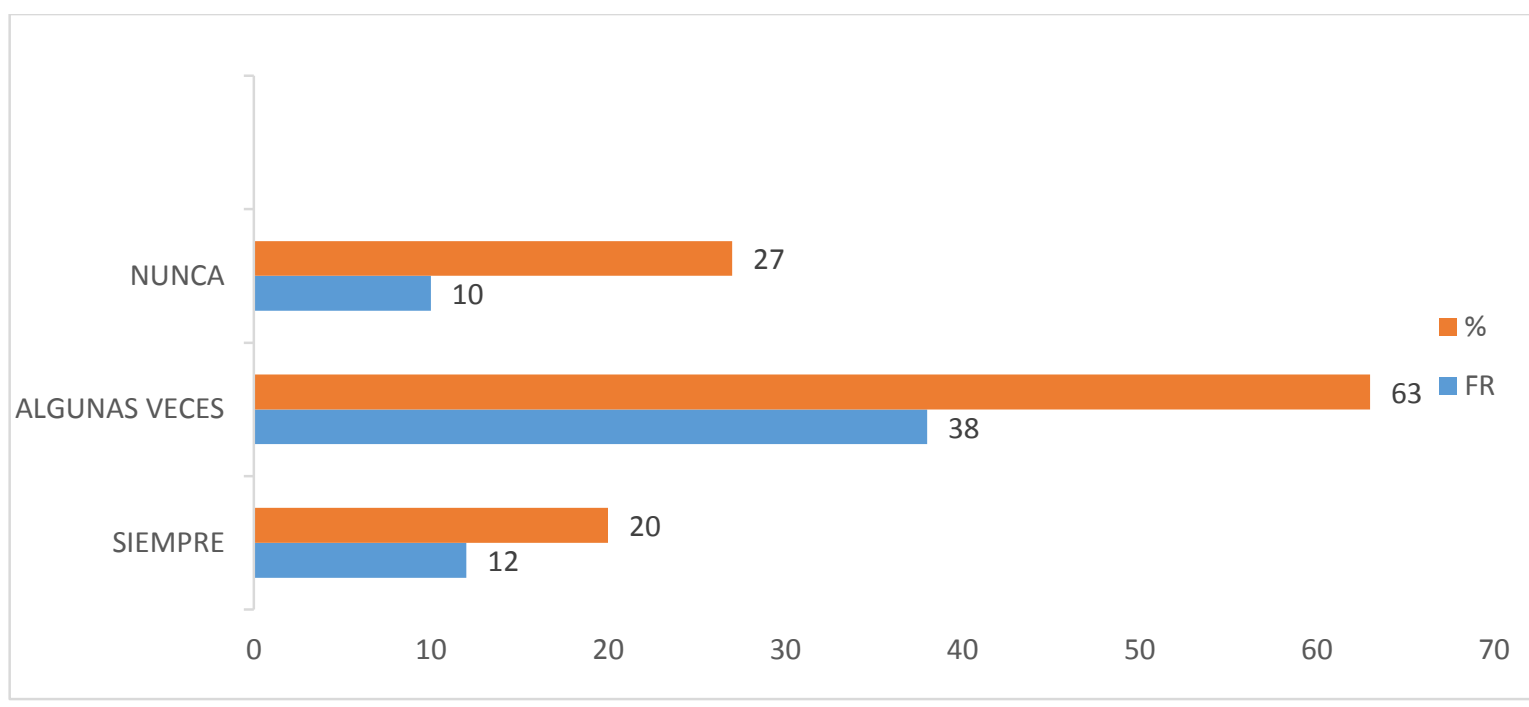

Grafico $\mathbf{N}^{\mathbf{0}} 3$. Distribución frecuencial y porcentual del indicador utilización de la música

Fuente: Elaboración propia (2019).

En la tabla y gráfico $\mathrm{N}^{\mathrm{o}} 3$, se presenta la distribución frecuencial y porcentual del indicador utilización de los mapas mentales como técnica del aprendizaje acelerado en la enseñanza de las estadísticas, en la que se evidencio que el $63 \%$ de los investigados coincidieron en opinar que solo algunas veces se usa la técnica de los mapas conceptuales para facilitar la organización con facilidad de los pensamientos y uso de forma máxima de sus capacidades mentales durante el desarrollo de las clases 
de Estadística. Seguido del $20 \%$ que afirmaron que si la utilizan y solo el $17 \%$ del total de las repuestas se indicaron para la alternativa nunca.

Dichos resultados, permite indicar que la expresión de pensamiento irradiante, es decir el de moverse en diversas direcciones a partir de un centro determinado, es decir de aquellos procesos de pensamiento asociativos que proceden de un punto central o se conectan con él, que en el caso de la enseñanza de las estadísticas son de gran importancia para lograr entender el avance de los contenidos programáticos del área.

\section{Conclusiones}

Posterior a los resultados presentados, y atendiendo el objetivo del artículo que permitió evaluar las técnicas del aprendizaje acelerado en la enseñanza de las estadísticas, se pudo concluir:

-La técnica del aprendizaje acelerado de relajación y atención, algunas veces logra crear en cada uno de los estudiantes un estado de gran receptividad para recibir de forma fácil todos los conocimientos que en materia de estadística son necesario para entender cada uno lograr e la compleja actividad diaria del quehacer académico.

-Se evidencio, que algunas veces la música como técnica del aprendizaje acelerado en la enseñanza de las estadísticas, sirve como medio para equilibrar la mente racional y la emocional. Entrar dentro de ellos mismos, hacerlos radicalmente interiores, despertando lo mejor, lo más noble de su vida a través de la música consolidan el amor a las personas y al trabajo que realizan como medio para alcanzar su proyecto de vida.

-Por otro lado, se pudo constatar que solo algunas veces se usa la técnica de los mapas conceptuales para facilitar la organización con facilidad de los pensamientos y uso de forma máxima de sus capacidades mentales durante el desarrollo de las clases de Estadística. Por otro lado, los docentes a partir del mapa conceptual pueden incluir una considerable cantidad de información de la materia, de forma visual en una sola página, facilitan la comprensión de las relaciones existentes entre los conceptos e ideas que se manejan en la materia. 


\section{Referencias Bibliográficas}

Buzan, T (2004). Como crear Mapas Mentales.- 1ª . Edición.- Ediciones Urano,

S. A.- España.

Cervantes, V (1999). El ABC de los Mapas Mentales.- 3ª . Edición.-

Asociación de Educadores Iberoamericanos, México.

Drapeau (1996). Aprendizaje Acelerado: Aprender a Aprender.- 1ª. Edición.- Editorial Océano.México.

Hernández, Fernández y Baptista (2014). Metodología de la Investigación. Tercera Edición. México .Editorial Me Graw Hill.

Pisano, B. (2000). La relajación y otras estrategias del superaprendizaje y su valor trascendental en el aula. México. Editorial Trillas

Pulpillo, M. (2001). Aprendizaje Total. España. Editorial .Kapeluz. 